\title{
Análise do Uso do Eletroencefalograma e do Índice Bispectral na Unidade de Terapia Intensiva
}

\author{
Analysis of the Electroencephalogram and the Bispectral Index Use in the Intensive Care Unit
}

\section{Ronaldo Bezerra de Queiroz", Maurus Marques de Almeida Holanda², Ana Karina Holanda Leite Maia ${ }^{3}$, Liana Clébia Soares Lima de Morais 4}

\begin{abstract}
RESUMO
Este trabalho revisa as atuais e futuras aplicaçóes da eletrencefalografia contínua (EEGc) e do índice bispectral para monitorar a função e fisiologia cerebral de pacientes com patologia neurológica aguda grave em unidade de terapia intensiva (UTI). Em pacientes comatosos a EEGc pode fornecer informação inalcançável por outros métodos, influenciar a administração terapêutica, além de ajudar a determinar o prognóstico de pacientes com lesão cerebral aguda. Esta técnica é a mais indicada para detecção de crises convulsivas subclínicas, que podem frequentemente ocorrer durante ou depois do tratamento do estado de mal epiléptico e depois de muitos tipos de lesóes cerebrais agudas, particularmente o trauma. EEGc pode detectar isquemia cerebral focal, tal como aquela causada por vasoespasmo em consequência da hemorragia subaracnóidea, tanto como isquemia global relacionada com a elevação da pressão intracraniana e pressão de perfusão cerebral insuficiente. Outras potenciais aplicaçóes incluem prognóstico do coma, mensuração da terapia sedativa continuamente infundida.
\end{abstract}

Unitermos. EEG, Eletroencefalograma, Eletroencefalografia, UTI, Cuidados intesivos, Índice Bispectral.

Citaçáo. Queiroz RB, Holanda MMA, Maia AKHL, Morais LCSL. Análise do Uso do Eletroencefalograma e do Índice Bispectral na Unidade de Terapia Intensiva.

\begin{abstract}
This paper reviews the current and future applications of continuous electroencephalography (cEEG) and bispectral index to monitor brain function and physiology of patients with acute neurological disease in the intensive care unit (ICU). In comatose patients, cEEG may provide otherwise unobtainable information and influence therapeutic management, and also help determine the prognosis of patients with acute brain injury. This technique is best used for the detection of subclinical seizures, which may frequently occur during or after treatment of convulsive status epilepticus and after many types of acute brain injury, particularly trauma. The other main application of cEEG is as a primary monitor of brain function. cEEG can detect focal cerebral ischemia, such as that caused by vasospasm after subarachnoid hemorrhage, as well as global ischemia related to intracranial pressure elevation and insufficient cerebral perfusion pressure. Other potential applications include prognostication in coma and titration of continuous infusion sedative therapy.
\end{abstract}

Keywords. EEG, Electroencephalogram, Electroencephalography, Intensive Care Unit, Intensive Care, Bispectral Index.

Citation. Queiroz RB, Holanda MMA, Maia AKHL, Morais LCSL. Analysis of the Electroencephalogram and the Bispectral Index Use in the Intensive Care Unit.
Trabalho realizado na UFPB, Joáo Pessoa-PB, Brasil.

1. Médico Neurologista do Hospital Universitário da Universidade Federal da Paraíba (UFPB), Mestre em Produtos Naturais e Sintéticos Bioativos - Farmacologia, João Pessoa-PB, Brasil.

2. Médico Neurocirurgiāo, Doutor em Produtos Naturais e Sintéticos Bioativos - Farmacologia, Professor de Neurologia da UFPB, João Pessoa-PB, Brasil. 3. Farmacêutica, Doutoranda do Programa de Pós Graduaçáo em Produtos Naturais e Sintéticos Bioativos - Farmacologia da UFPB, Professora de Microbiologia da FAMENE (Faculdade de Medicina Nova Esperança), João Pessoa$\mathrm{PB}$, Brasil.

4. Farmacêutica, Doutora em Produtos Naturais e Sintéticos Bioativos - Farmacologia, Professora de Fisiologia da UFPB, João Pessoa-PB, Brasil.
Endereço para correspondência: Prof. Dra. Liana Clébia Soares Lima de Morais Rua Alzira Coutinho de Araújo, 95, Apt. 401 - Bancários CEP 58051-119, João Pessoa-PB, Brasil. E-mail: lianaltf@hotmail.com 


\section{INTRODUÇÃO}

Diversos estudos relatam que o EEG era realizado primariamente em unidades de acompanhamento de epilepsia para classificação e quantificação das crises, identificação das descargas epileptiformes interictais, avaliação pré-cirúrgica de candidatos a cirurgia para epilepsia ${ }^{1,2}$.

Nos últimos anos, uma série de evoluçóes tecnológicas foi introduzida no ambiente da unidade de terapia intensiva (UTI). Dentre tais inovaçóes, merece destaque a monitorização da função cerebral, a qual tem se tornado cada vez mais disponível, sendo de grande importância especialmente no contexto das doenças neurológicas agudas, principalmente nos pacientes comatosos, oferecendo significativas opções de tratamento ${ }^{3}$.

No decorrer de um acompanhamento neurológico em UTI, é possível fazer detecção precoce de complicações secundárias, bem como ter condiçōes de realizar uma avaliação contínua dos efeitos das intervenções terapêuticas. Merece relevância este aspecto, posto que, após uma lesão cerebral aguda, o surgimento de agressões secundárias aumenta substancialmente a morbidade e a letalidade em uma ampla série de pacientes com injúria cerebral ${ }^{4}$.

Alteraçóes significativas da disfunção ou da estrutura cerebral podem não ser identificadas inicialmente no exame clínico neurológico. Dessa forma, uma deterioração da atividade cerebral ou uma melhoria do quadro podem passar despercebidas. Portanto, a capacidade para avaliar deterioração ou melhora neurológica, de importância clínica, em pacientes graves necessitando de cuidados intensivos tem sido bastante limitada. A deterioração cerebral, por exemplo, pode tornar-se evidente somente no aparecimento de evento grave e irreversível. Esta dificuldade tem estimulado o desenvolvimento de novas ferramentas clínicas de avaliação ${ }^{4}$.

Experiências clínicas recentes e dados da literatura sugerem o uso do eletroencefalograma (EEG) contínuo e de outras técnicas de monitorização que podem melhorar consideravelmente a avaliação neurológica e cuidados dos pacientes críticos, como também ajudar a determinar o prognóstico destes pacientes ${ }^{4}$.

O EEG digitalizado pode ser matematicamente transformado pelo processo conhecido como análise de Fourier, que separa o complexo sinal do EEG em vários componentes de onda, ou seja, em cada porção de di- ferente amplitude, mas cuja soma corresponde à forma original da onda. Com o emprego deste método, surgem vários parâmetros de avaliação 5 .

O índice bispectral (BIS) é um parâmetro multifatorial derivado do eletroencefalograma que permite a monitorização do componente hipnótico da anestesia. Foi obtido a partir de algoritmo derivado da análise de grande número de eletroencefalogramas de voluntários e pacientes submetidos a sedaçóes e anestesia geral com diferentes agentes ${ }^{6}$. Recentemente foi introduzido em $\mathrm{UTI}^{7}$.

Com o advento do EEG digital tornou possível a sua utilização para monitorização em pacientes graves em UTI pela possibilidade da gravação do exame, uso de monitores em vários pacientes, como também o uso da internet no envio destes dados para uma análise à distância, bem como a compactação e decodificação destes dados através de programas de computadores trazendo uma melhor interpretação destes, na forma de gráficos ou figuras (EEG quantitativo $)^{4,8}$.

Desse modo, no momento da realização da leitura do EEG, é possível mudar os filtros, avaliar por meio de várias montagens, ou mesmo ajustar a sensibilidade para melhor interpretação e conclusão. Portanto, o EEG está se tornando cada vez mais aceito como parte do rol de técnicas de vigilância utilizadas em UTI ${ }^{4}$.

Fica claro a existência de uma lacuna na avaliação contínua da fisiologia cerebral quando comparada com a monitoração dos sistemas cardiovascular e pulmonar ${ }^{1}$.

O BIS utilizado com a finalidade de avaliar o grau de sedação em anestesia é comparado, na literatura pertinente, com escalas de avaliação clínica de sedação, mas seu uso em UTI demonstra ainda carência de estudos ${ }^{6,9}$.

Portanto, este trabalho revisa as atuais e futuras aplicações da eletrencefalografia contínua e do índice bispectral para monitorar a função e fisiologia cerebral de pacientes com patologia neurológica aguda grave em unidade de terapia intensiva.

\section{MÉTODO}

A metodologia utilizada para este estudo consistiu de uma revisão bibliográfica de artigos publicados recentemente, em periódicos nacionais e internacionais, a partir do ano de 1997 até 2010 relacionados com o uso 
do eletroencefalograma contínuo e do índice bispectral (BIS), em unidades de terapia intensiva. Sendo incluída a pesquisa na U.S. National Library of Medicine (PubMed) e na Biblioteca Virtual em Saúde (BVS - BIREME). Outros materiais foram obtidos por meio de pesquisa nãoestruturada com consulta a livros-texto consagrados na área de UTI relacionados ao foco da pesquisa. A pesquisa incluiu artigos em espanhol, inglês e português. Utilizando-se os unitermos EEG, eletroencefalograma, eletroencefalografia, UTI, cuidados intesivos, foram encontrados vários artigos, porém, apenas 37 relacionados com monitorização em UTI, sendo utilizados 24 neste manuscrito por serem mais recentes.

\section{RESULTADOS}

\section{MONITORIZAÇÃO NEUROLÓGICA INTENSIVA}

A necessidade da monitorização neurológica na UTI é a da prevenção ou o diagnóstico precoce dos eventos que podem desencadear lesôes cerebrais secundárias ou agravar as lesões já existentes. Esta monitorização é multimodal devido às limitaçóes das diferentes monitoraçóes e desta forma tem que se monitorizar diversas variáveis ao mesmo tempo ${ }^{7}$. Que inclui a avaliação clínico neurológica ${ }^{7,10}$, pressão intracraniana $(\mathrm{PIC})^{11}$, pressão de perfusão cerebral $(\mathrm{PPC})^{7,11}$, capnometria ${ }^{7}$, oximetria do bulbo jugular ${ }^{7,11}$, tomografia computadorizada (TC) $)^{7,11}$, monitorização da temperatura cerebral ${ }^{7,11}$, monitorização do $\mathrm{PO}_{2}$ cerebral ${ }^{10}$, microdiálise ${ }^{12,13}$, doppler transcraniano $(\mathrm{DTC})^{7,10,11}$.

\section{Índice Bispectral (BIS)}

$\mathrm{O}$ aparelho de BIS possui um adesivo que contempla as derivaçóes na regiáo frontal do crânio idênticas ao do eletroencefalograma (EEG). Através de uma constante matemática as informaçóes sobre ondas alfa e beta e teta nestas derivaçóes são convertidas em uma escala numérica de 0 a 100 (100 desperto / 0 coma = EEG isoelétrico $)^{14}$.

O BIS é uma medida objetiva de sedação tradicionalmente usados em anestesia geral, já está sendo usado em cuidados intensivos. Monitores BIS fornecem informaçôes clinicamente relevantes para o ajuste de doses de medicaçôes sedativas. A utilização de um monitor BIS, que é um dispositivo náo invasivo, pode afetar os custos globais de sedação do doente em estado crítico. Ele fornece um índice do grau de sedação em pacientes em ventilação mecânica recebendo agentes de sedação após o trauma, cirurgia ou acompanhamento de paciente crítico. O BIS foi desenvolvido inicialmente como um método de monitorização adjuvante da anestesia durante a cirurgia. Monitores BIS foram desenvolvidos por analisar certas características do EEG (análise bispectral), observando vários pontos finais para a consciência, e incorporando funcionalidades que se correlacionam melhor com as mudanças no estado hipnótico. Um monitor BIS prevê um processo contínuo de exibição do BIS atual e vários parâmetros importantes para acompanhamento (Figura 1). O BIS é exibido no canto superior esquerdo do monitor. Esta pontuação varia de 0 a 100 e é uma medida da atividade elétrica cerebral.Uma pontuação de 90 a 100 correlaciona-se com um estado de vigília, pontuações de 70 para 80 indica sedação, escores de 60 a 70 sedação profunda, e escores de 40 a 60 esta presente na anestesia geral (Figura 2). Um canal único mostra o traçado do EEG continuamente. O índice do sinal de qualidade (QSI) é uma barra que indica a confiabilidade do sinal, quanto maior o QSI, mais confiável é o BIS exibido. A barra eletromiografia (EMG) indica a atividade da EMG, o que reflete a estimulação muscular e pode ser alterado por qualquer coisa que aumente tônus muscular ou movimento do músculo. Algumas das principais causas de EMG aumentada em um monitor BIS: se ocorrer atividade motora, dor, atividade de apreensão,movimentos dos olhos, e contato deficiente do eletrodo na pele. Quanto maior a atividade do EMG, menor é a confiabilidade do BIS. A razão de supressão indica a porcentagem do traçado isoelétrico do EEG. A parte da tela de Orientação geral mostra o histórico de vários parâmetros ${ }^{14}$.

A monitorização neurológica com BIS é muito útil para acompanhar pacientes neurológicos críticos sob uso de sedação, informando quando se atingiu o estágio de surtos/supressão, permitindo a titulação da dose do barbitúrico. Contudo, como ele utiliza apenas as derivaçóes frontais, observam-se algumas dificuldades para pacientes com traumas cranianos difusos ou áreas isquêmicas em núcleos profundos. Em 1996, o FDA (Food and Drug Administration) recomendou o uso do BIS para monitorização da profundidade da anestesia com o objetivo de reduzir a ocorrência de despertar e memória intra-operatória. 


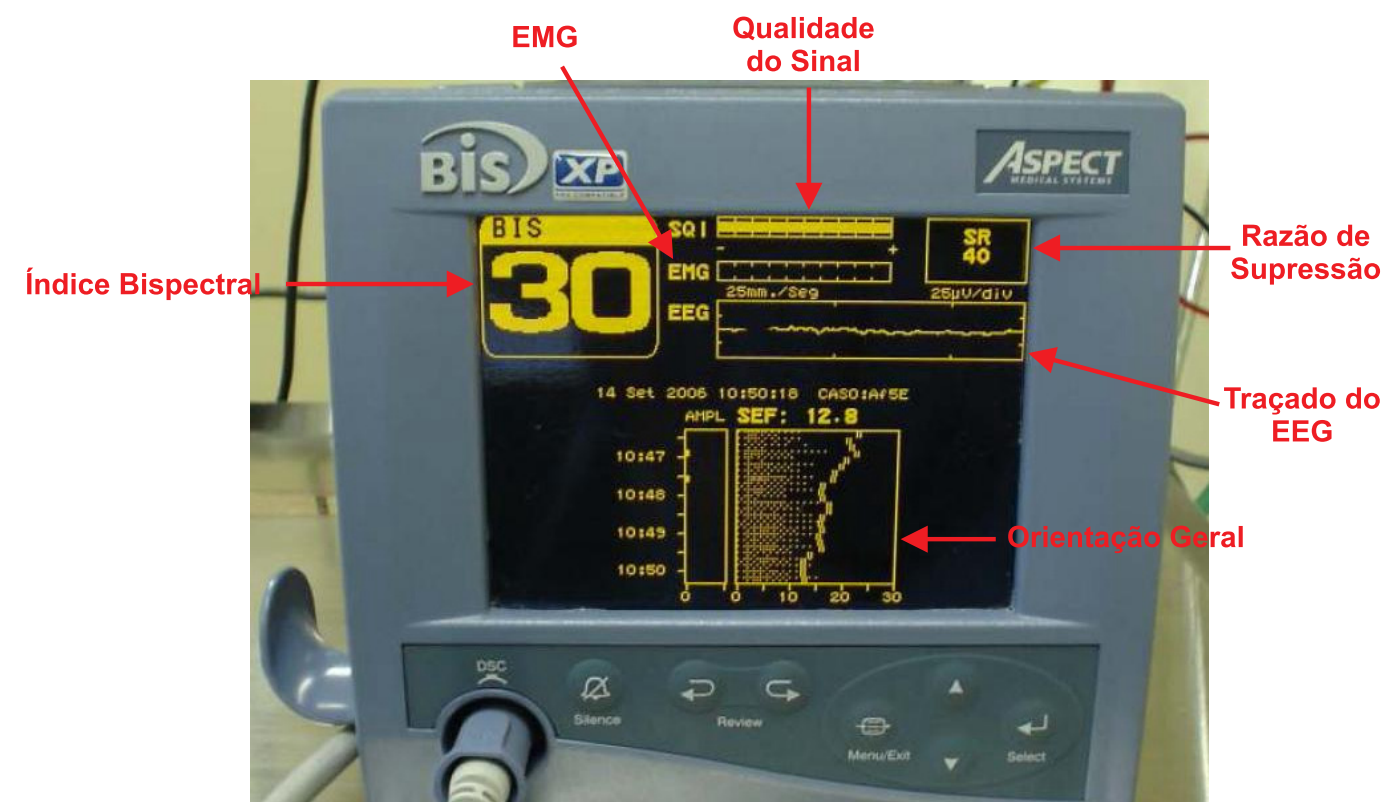

Figura 1. Monitor do índice bispectral com componentes chaves.

Permite também despertar mais rápido da anestesia e permanência mais curta na sala de recuperação pós-anestésica, o que, em última análise, poderá reduzir os custos do procedimento anestésico-cirúrgico ${ }^{15}$.

O BIS pode apresentar valores falsamente elevados ou reduzidos, decorrentes de condiçóes do paciente ou açôes de anestésicos não previstos quando da elaboração do seu algoritmo. Estas situaçóes deverão ser reconhecidas pelo anestesiologista a fim de evitar conduta equivocada no manuseio da anestesia ${ }^{6}$.

Existem algumas pesquisas sobre tentativas do seu uso para outras situações além de controle de sedação, com resultados interessantes, porém em análise para confirmação e validação clínica ${ }^{14}$.

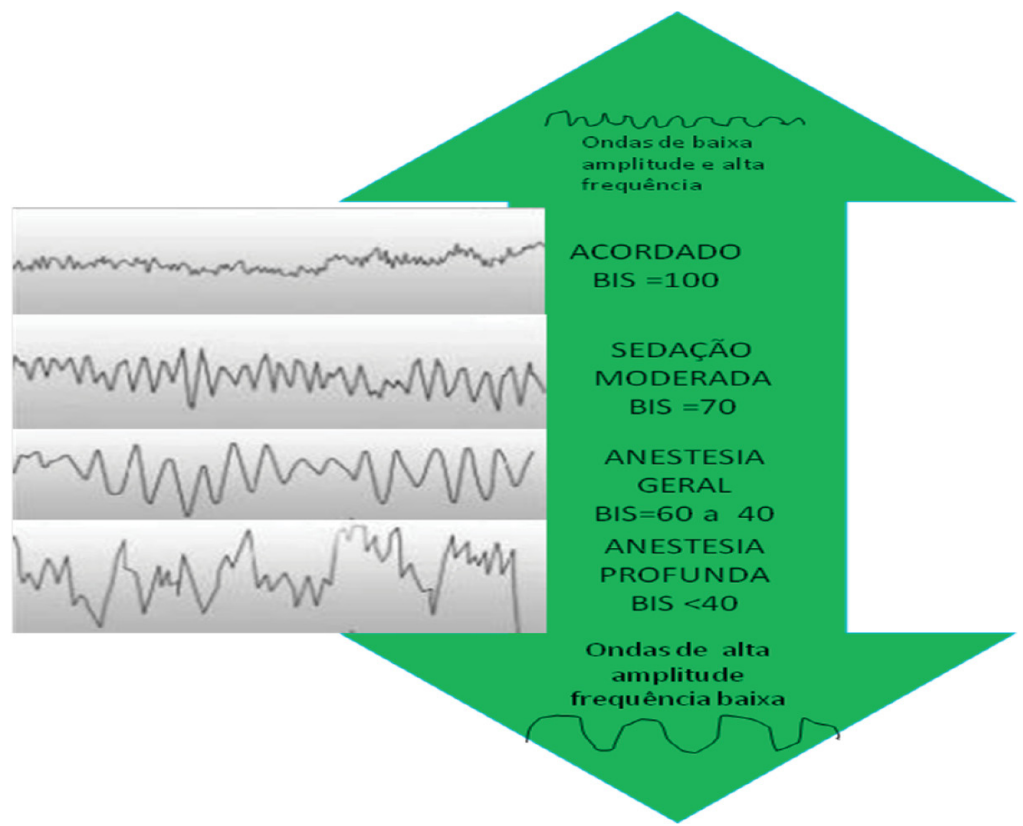

Figura 2. Correlação entre as ondas corticais e o estado clínico.

\section{Eletroencefalografia Contínua (EEGc)}

A monitorização contínua com EEG é uma técnica não invasiva que promove uma avaliação dinâmica sobre a função cerebral que permite detectar precocemente alteração do estado neurológico, especialmente quando o exame clínico está limitado, como nos casos de doentes comatosos críticos e que podem ter efeitos negativos múltiplos com aumento da lesão cerebral. A indicação da monitorização contínua com EEG na unidade de terapia intensiva inclui: a detecção de crises não convulsivas ou estado de mal epiléptico (estado epiléptico) não convulsivo (EENC), o controle do estado de mal epiléptico (estado epiléptico) com o tratamento intravenoso de drogas antiepilépticas, a detecção de isquemia cere- 
bral tanto no vasoespasmo pós hemorragia subaracnóidea como em outros pacientes com elevado risco de acidente vascular cerebral isquêmico e ajudar no prognóstico de pacientes comatosos tanto no acompanhamento de casos de pós parada cardíaca como nas lesóes cerebrais agudas ${ }^{1}$. Há uma ocorrência maior de crises não convulsivas em pacientes com alteração do estado mental com história pregressa de epilepsia, flutuação do nível de consciência, história de lesão cerebral aguda, recente estado epiléptico, atividade estereotipada como movimentos paroxísticos, nistagmo e espasmos corrobora para a indicação da EEGc. A monitorização da terapia, que está sendo feita no coma induzido por elevação da pressão intracraniana, no estado epiléptico refratário e na avaliação do nível de sedação (coma barbitúrico) também são indicações da $\mathrm{EEGc}^{16}$.

\section{APLICAÇÓES DA EEGc RELATADAS NA LITERA- TURA PERTINENTE}

\section{Detecção de Crises Não Convulsivas e Estado Epilép- tico Náo Convulsivo}

Crises não convulsivas (CNC) e EENC são cada vez mais reconhecidas como ocorrências comuns em UTI, onde 8 a $48 \%$ dos pacientes comatosos podem ter CNC dependendo do estudo realizado. No estudo de pacientes de todas idades com inexplicável decréscimo do nível de consciência ou suspeita de crises subclínicas 19\% tiveram qualquer tipo de crise $^{17}$.

CNC são crises eletrográficas com pouca ou nenhuma manifestação clínica. Sendo assim o EEG é necessário para sua detecção. EENC ocorre quando CNC são prolongadas. Segundo a definição mais comum, EENC são crises eletrográficas de pelo menos trinta minutos de duração. Apesar de que algumas formas de CNC serem passíveis de ocorrer em pacientes ambulatoriais, que podem apresentar certa confusão mental, mas, nos pacientes de UTI a manifestação mais comum é a depressão do nível de consciência ${ }^{18}$.

As etiologias para CNC e EENC em pacientes de UTI são semelhantes às causas de crises convulsivas nestes pacientes. Estes incluem lesôes estruturais agudas, infecções, distúrbios metabólicos, tóxicos, epilepsia, todos os diagnósticos comuns no paciente crítico ${ }^{16}$. No entanto, CNC é a mais comum manifestação em pacientes in- ternados na UTI e deve ser considerado como uma das causas do estado mental alterado, especialmente em populaçóes de alto risco como por lesão cerebral traumática, infecção do sistema nervoso central, hemorragia intracerebral, hemorragia subaracnóidea, acidente vascular cerebral isquêmico ${ }^{17}$.

\section{a) Estado Epiléptico}

Em muitos pacientes que apresentam estado epiléptico, crises eletrográficas podem persistir mesmo quando a atividade convulsiva tenha cessado. Na maioria destes pacientes o coma foi a única manifestação clínica. Pacientes com EENC depois do estado epiléptico tem mais do que duas vezes maior mortalidade comparada com pacientes que finalizaram as crises quando cessaram atividade convulsiva. Assim, EEGc deveria ser executada em qualquer paciente que não recuperasse rapidamente a consciência depois de uma crise convulsiva com finalidade de detectar uma atividade convulsiva. Isto inclui pacientes que estáo sedados e ou paralisados durante o tratamento do estado epiléptico com um nível de consciência que não pode ser adequadamente avaliada ${ }^{8}$.

\section{b) Hemorragia Subaracnoidea}

Convulsões têm sido encontradas após hemorragia subaracnóidea causada por aneurisma. Em um estudo com mudança do estado mental em 19\% dos pacientes com hemorragia subaracnóidea monitorizados tiveram crises convulsivas ${ }^{18}$. A maioria destas crises foram CNC. Crises convulsivas depois de hemorragia subaracnóidea podem piorar a lesão cerebral, como ambas convulsivas e CNC estão associadas com piores resultados nestes pacientes ${ }^{8}$.

\section{c) Hemorragia Intracerebral}

A presença de sangue pode provocar crises convulsivas no cérebro lesado porque o índice de crises convulsivas é maior em pacientes com sangramento intraparenquimatoso traumático e não traumático do que naqueles com infarto brando. CNC foram associadas com o desvio da linha média do cérebro associada com a tendência a piora dos resultados, como também o aumento do volume da hemorragia. Em adiçáo, autores encontraram que descargas epileptiformes periódicas (DEP) são um indi- 
cativo independente de resultado insatisfatório. Os trabalhos demonstram que a EEGc detecta CNC e permite tratamento $^{19}$.

\section{d) Acidente Vascular Cerebral Isquêmico (AVCI)}

No estudo série Columbia, 11\% dos 56 pacientes com isquemia cerebral submetidos à EEGc tiveram convulsões; estas foram CNC em todos os pacientes ${ }^{17}$. Muitos estudos têm mostrado que crises convulsivas agudas estão associadas com o aumento da mortalidade em pacientes com AVCI. A associação entre $\mathrm{CNC}$ e resultados pós AVCI é desconhecida. Entretanto em modelos animais de AVCI utilizando roedores, CNC foram associada com o aumento do volume do infarto e triplicou a mortalidade ${ }^{8}$.

\section{e) Traumatismo Crânio Encefálico (TCE)}

Crises convulsivas precoces são de comum ocorrência depois de TCE. Por causa desta frequência, o uso de profilaxia para crises convulsivas depois de um TCE tem tornado menos comum convulsóes agudas. Poucos estudos têm examinado a incidência de CNC pós TCE. No estudo de 96 pacientes com moderado ou severo TCE submetidos à EEGc foram encontrados 22 pacientes que tiveram convulsão, e a metade tiveram somente $\mathrm{CNC}^{19}$. No estudo série Columbia, $18 \%$ de 51 pacientes com TCE monitorados com EEGc tiveram convulsóes, todos eles tiveram $\mathrm{CNC}$, e $8 \%$ tiveram $\mathrm{EENC}^{18}$. O número relativamente baixo de crises convulsivas nestas séries é possivelmente devido a rotina do uso de anticonvulsivante profilaticamente depois do TCE, mas a ocorrência de $\mathrm{CNC}$ nestes pacientes sugerem que a profilaxia não deve ser suficiente para alguns pacientes. A exatidão da relação entre convulsão e resultado ainda não é clara, especialmente como convulsôes precoces são altamente correlacionadas com lesão severa. Alguns estudos mostram como convulsão precoce é um fator de risco para piores resultados com TCE severo. Os estudos não examinaram entretanto CNC tendo similar impacto ${ }^{8}$.

\section{f) Pacientes no Pós-Operatório}

Procedimentos neurocirúrgicos especialmente aqueles que envolvem lesóes supratentoriais estấo associados com um risco de 4 a 17\% de convulsóes no pós-ope- ratório. Quando tem história de epilepsia anteriormente a cirurgia este risco sobe para 34\%. Pouco é conhecido de $\mathrm{CNC}$ nestes pacientes ${ }^{18}$. Convulsóes no pós-operatório não estão limitadas aos procedimentos neurocirúrgicos. Elas podem ocorrer em qualquer pós-operatório onde haja risco de distúrbio metabólico, injúria neurológica aguda ou relato de droga neurotóxica. Um particular risco são os pacientes transplantados. As convulsões são comuns no pós-operatório imediato de pâncreas, fígado, pulmão, coração, rim e transplante de medula óssea. Nas cirurgias cardíacas o risco de desenvolver lesão neurológica aguda como AVC ou hipóxia pode predispor a convulsão durante a cirurgia ou no pós-operatório. A incidência de CNC e EENC nestes pacientes não têm sido estudadas. Entretanto, como em outros pacientes críticos com aumento do risco de convulsóes, CNC deveria ser considerada como uma causa para contribuir para uma inexplicável mudança do estado mental em pacientes transplantados ${ }^{8}$.

\section{g) Injúria Hipóxica - Isquêmica}

Após parada cardíaca é comum o surgimento de convulsóes. Em pacientes comatosos por hipoxia/anoxia/ isquêmica foi encontrado taxa de EENC e CNC em 20\% deles ${ }^{17}$. A presença de convulsáo pós parada cardíaca pode ter importante implicação no prognóstico. A EEGc pode ser importante ferramenta na identificação de CNC em pacientes pós parada cardíaca que apresentam tremores durante principalmente o reaquecimento depois da hipotermia usada nestes casos para neuroproteção ${ }^{8}$.

\section{h) Encefalopatia Tóxico-Metabólica}

Pacientes críticos clínicos e cirúrgicos são susceptíveis a anormalidades tóxicas, eletrolíticas e metabólicas que causa tanto alteração do estado mental como convulsóes. Claassen et al. em 2004 encontrou 21\% de CNC em pacientes com o diagnóstico primário neurológico de encefalopatia tóxico-metobólica (hiponatremia, hipo ou hiperglicemia, hipocalcemia, retirada ou intoxicação por drogas, uremia, insuficiência hepática, encefalopatia hipertensiva e sepse).

\section{Duração da Monitorizaçáo para CNC}

Estudos que incluíram pacientes que estão em es- 
tado de coma em qualquer UTI, UTI pediátrica, ou ter inexplicáveis alteraçóes do estado mental em qualquer lugar do hospital, têm sido encontradas taxas de CNC entre $8 \%$ e $37 \%$, sugerindo que pode ser encontrada em qualquer paciente de risco.

CNC são claramente comuns em pacientes críticos, mas, a evidência que elas piorem os resultados e requerem pronta identificação e tratamento os estudos são controversos. Náo houve um estudo prospectivo controlado para determinar se o tratamento de CNC e EENC melhorem os resultados neurológicos.

Quanto ao tempo de monitorizaçáo com EEGc para o diagnóstico de $\mathrm{CNC}$ em pacientes não comatosos é provável que 24 horas de monitorização seja suficiente para excluir CNC, mas períodos maiores possam ser requeridos para pacientes comatosos ${ }^{8,20}$.

\section{Detecção de Isquemia}

Uma aplicação antiga da monitorização com EEG contínuo que é a detecção de isquemia cerebral tem recebido renovado interesse devido a associação de atividade neuronal e fluxo sanguíneo cerebral (FSC), porque mudanças no EEG ocorrem dentro de segundos de redução do FSC e isto é a base da monitorização contínua com EEG durante o ato operatório de pacientes submetidos a cirurgia com alto risco de isquemia cerebral como a endarterectomia carotídea. Assim, a EEGc pode detectar uma janela em que uma intervenção pode prevenir um lesão cerebral permanente. Isto se torna importante como trombolíticos e terapias endovascular tem se mostrado ser efetivas no AVCI agudo e vasoespasmo, especialmente quando o tratamento é instituído precocemente. O EEG não se altera com o aumento da pressão intracraniana (PIC) até que haja comprometimento do FSC $^{8}$.

\section{Verificação da Eficácia Terapêutica}

A EEGc tem seu uso especial na avaliação da resposta em intervençôes que visem uma redução da atividade neurológica para diminuir o metabolismo cerebral. Pode informar sobre o nível de sedação nos pacientes críticos especialmente nos estados de bloqueio neuromuscular. Serve como guia no tratamento do estado epiléptico com infusão intravenosa de anestésicos, tais como midazolam, propofol ou pentobarbital. Também pode ser útil a
EEGc no ajuste na taxa de infusão para manter supressão suficiente enquanto minimiza os efeitos adversos das drogas como hipotensão, comprometimento da função renal e hepática principalmente. Serve este exame, como guia na retirada de anestésicos destes pacientes como no coma farmacologicamente induzidos nos traumas de crânio ${ }^{8}$.

\section{Avaliação Prognóstica}

Monitorização com EEGc pode dar informaçóes prognósticas após TCE, podendo auxiliar médicos e familiares na tomada de decisões. Vários aspectos correlacionados com resultados após lesão cerebral aguda foram apresentados na detecção de CNC e EENC anteriormente. A arquitetura anormal do sono, descargas epileptiformes, perda de reatividade encontradas na monitorização com EEGc pode ser determinante para o prognóstico do paciente comatoso. Isto não é claro se eles são marcadores da lesão cerebral ou se eles causarão danos futuros. Após parada cardíaca supressão generalizada, padrão surtosupressão com atividade epileptiforme generalizada, ou complexos periódicos generalizados tem sido fortemente associado com mau resultado ${ }^{17}$. O EEG, com seus principais achados, foi capaz de diferenciar as diversas taxas de sobrevivências em uma amostra estudada, constituindose, portanto, bom instrumento de prognóstico para pacientes atendidos nas unidades de emergência hospitalar ${ }^{21}$.

\section{DISCUSSÃO}

A indicação clínica para monitorização contínua, é indicada sempre o mais cedo possível na detecção de um processo fisiopatológico, para que possa ser impedida as suas consequências duradouras, tanto em adultos como em crianças. Os principais eventos reversíveis, que podem ser captados pela monitorização da EEGc são: status epilepticus (SE) não-convulsivo; as consequências neurológicas do aumento da pressão intracraniana; mudanças no EEG consequentes de modificaçóes no grau de encefalopatia hepática e/ou influências de drogas; alteraçóes do EEG que são induzidos pela isquemia de penumbra ${ }^{22}$.

O papel exato e o custo-eficácia da EEGc no momento atual permanecem obscuros. Entretanto, ela tem um potencial significativo para aperfeiçoar resultados neurológicos em uma variedade de situaçóes. Ressalte-se que, quando o EEG contínuo é combinado com uma si- 
tuação individualizada para cada caso, tomando decisóes com base na fisiologia via monitorização multimodal do cérebro, intensivistas podem identificar quando o cérebro está em risco de lesão ou injúria neuronal já presente e podem intervir antes que haja uma lesão cerebral definitiva. É o melhor método para se detectar atividade convulsiva ${ }^{23}$.

A obtenção de EEGc com alto padrão em sua gravação na UTI persiste como um desafio, sendo necessário a idealização de uma técnica adequada para conectar os pacientes e manter esta conexão 24 horas por dia, além da obtenção de cabos que sejam compatíveis com realização de ressonância nuclear magnética e tomografia, cabos estes, já disponíveis comercialmente nos Estados Unidos ${ }^{17}$.

Em adição, é certo que o paciente de UTI precisa ser reposicionado com frequência, sendo difícil manter a integridade dos eletrodos. Além disso, a indisponibilidade de programas mais simples, torna ainda necessária a presença do eletroencefalografista para revisar os EEG gravados.

Taher e colaboradores apresentaram em dezembro de 2010 na 64ª Reuniāo Anual da Sociedade Americana de Epilepsia a confirmação da importância do EEGc na unidade de terapia intensiva como ferramenta de diagnóstico na detecção de crises não convulsivas em comparaçấo com outros métodos ${ }^{24}$.

Quanto ao uso do BIS na unidade de terapia intensiva faz-se necessário estimular a investigação e o debate no sentido de aumentar a compreensão dos benefícios e limitações do uso de monitores BIS em cuidados intensivos. Existindo uma carência de estudos com este aparelho e validação clínica ${ }^{14}$.

A pesquisa da literatura, deixa claro que existem ainda muitos obstáculos para a efetiva monitorização da função cerebral em tempo real, de forma confiável, prática e amplamente disponível. Entretanto, merece destaque o fato de que a tecnologia está progredindo rapidamente neste sentido.

\section{CONCLUSÃO}

Os valores do BIS devem ser entendidos como dado adicional na monitorização neurológica e interpretados à luz de outros achados clínicos e de outros monitores.
É coerente portanto, para que a monitorização com a EEGc seja largamente integrada à prática diária, há uma necessidade de se desenvolver um EEG quantitativo dotado de alarmes para questóes clínicas específicas, com programas que melhorem a detecção de artefatos e determine a importância de achados característicos no EEG. A terapêutica baseada nas evidências encontradas da EEGc podem melhorar os resultados em pacientes com lesão cerebral severa.

\section{REFERÊNCIAS}

1.Claassen J, Mayer SA. Continuous electroencephalographic monitoring in neurocritical care. Curr Neurol Neurosci Rep 2002; 2:534-40.

http://dx.doi.org/10.1007/s11910-002-0042-1

2.Costa VV, Torres RVSD, Arci ECP, Saraiva RA. Comparação dos valores do índice bispectral em pacientes com paralisia cerebral em estado de vigília. Rev. Bras. Anestesiol 2007; 57:382-90.

3.Scheuer ML. Portable remote wireless EEG review using a cellular CDMA network. Epilepsia 2001;42:27-8.

4.Scheuer ML. Continuous EEG monitoring in the intensive care unit. Epilepsia 2002; 43:114-27.

http://dx.doi.org/10.1046/j.1528-1157.43.s.3.7.x

5.Vianna PTG. Monitor de profundidade da hipnose: a eletroencefalografia bispectral. Rev Bras Anestesiol 2001;51:418-25.

http://dx.doi.org/10.1590/S0034-70942001000500008

6.Duarte LTD, Saraiva RA. When the bispectral index (bis) can give false results. Rev. Bras Anestesiol 2009; 59:99-109.

7.Feldman Z, Robertson CS. Upadate on neurologic critical care. Crit Care Clinics 1997;13:1-233.

8.Friedman D, Claassen J, Hirsch LJ. Continuous electroencephalogram monitoring in the intensive care unit. Anesth Analg J 2009; 109:506-23.

http://dx.doi.org/10.1213/ane.0b013e3181a9d8b5

9.Powers KS, Nazarian EB, Tapyrik SA, Kohli SM, Yin H, van der Jagt EW, et al. Bispectral Index as a Guide for Titration of Propofol During Procedural Sedation Among Children. Pediatrics 2005;115:1666-74.

http://dx.doi.org/10.1542/peds.2004-1979

10.Procaccio F, Stocchetti N, Citerio G, Berardino M, Beretta L, Della Corte F, et al. Guidelines for the treatment of adults with severe head trauma (part I). Initial assessment; evaluation and pre-hospital treatment; current criteria for hospital admission; systemic and cerebral monitoring. J Neurosurg Sci 2000;44:1-10.

11.Pereira GAJ, Coletto FA, Martins MA, Marson F, Pagnano RCL, Dalri MCB, et al. O papel da unidade de terapia intensiva no manejo do trauma. Rev Med Ribeirão Preto 1999; 32:419-37.

12.Blanco-Lezcano L, Pavón-Fuentes N, Blanco-Lezcano V. Microdiálisis cerebral. Principales aplicaciones de la técnica. Rev de Neurolog 2001;33:464-70. 13.Bellander BM, Cantais E, Enblad P, Hutchinson P, Nordström CH, Robertson $\mathrm{C}$, et al. Consensus meeting on microdialysis in neurointensive care. Intensive Care Med 2004; 30:2166-9.

http://dx.doi.org/10.1007/s00134-004-2461-8 
14. Olson DM, Chioffi SM, Macy GE, Meek LG, Cook HA. Potential benefits of bispectral index monitoring in critical care. A case study. Crit Care Nurse 2003; 23:45-52.

15. Olson DM, Graffagnino C, King K, Lynch JR. Toward solving the sedation-assessment conundrum: bi-spectral index monitoring and sedation interruption. Crit Care Nurs Clin North Am 2005;17:257-67. http://dx.doi.org/10.1016/j.ccell.2005.04.008

16.Chong DJ, Hirsch LJ. Which EEG patterns warrant treatment in the critically ill? Reviewing the evidence for treatment of periodic epileptiform discharges and related patterns. J Clin Neurophysiol 2005; 22:79-91.

http://dx.doi.org/10.1097/01.WNP.0000158699.78529.AF

17.Kurtz P, Hanafy KA, Claassen J. Continuous EEG monitoring: is it ready for prime time? Curr Opin Crit Care 2009;15:99-109.

http://dx.doi.org/10.1097/MCC.0b013e3283294947

18.Claassen J, Mayer SA, Kowalski RG, Emerson RG, Hirsch LJ. Detection of electrographic seizures with continuous EEG monitoring in critically ill patients. Neurology 2004;62:1743-51.

http://dx.doi.org/10.1212/01.WNL.0000125184.88797.62

19.Vespa P. Continuous EEG monitoring for the detection of seizures in traumatic brain injury, infarction, and intracerebral hemorrhage: "to detect and protect". J Clin Neurophysiol 2005;22:99-106.
http://dx.doi.org/10.1097/01.WNP.0000154919.54202.E0

20.Vespa PM, Nenov V, Nuwer MR. Continuous EEG Monitoring in the Intensive Care Unit: Early Findings and Clinical Efficacy. J Clin Neurophysiol 1999; 16:1-13.

http://dx.doi.org/10.1097/00004691-199901000-00001

21.Borges MA, Botós HJ, Bastos RF, Godoy MF, Marchi NS. Emergency EEG Study of survival. Arq Neuro-Psiquiatr 2010; 68:174-8.

http://dx.doi.org/10.1590/S0004-282X2010000200004

22.Guérit JM, Amantini A, Amodio P, Andersen KV, Butler S, Weerd A, et al. Consensus on the use of neurophysiological tests in the intensive care unit (ICU): Electroencephalogram (EEG), evoked potentials (EP), and electroneuromyography (ENMG). Neurophysiol Clin/Clin Neurophysiol 2009;39:71-83. http://dx.doi.org/10.1016/j.neucli.2009.03.002

23.Alcântara TFDL, Marques IR. Avanços na monitorização neurológica intensiva: implicaçōes para enfermagem. Rev Bras de Enferm 2009;62:894-900. 24.Taher Y, Evan F, Paige A, Politsky J, Lambrakis C, Laban O, Lee J, Lancman M. Analysis of Continuous Electroencephalography in the Intensive Care Unit for Nonconvulsive Seizure Activity- A Comparison of Four Methods. Citado Dez/ 2010. Estados Unidos, 64 Annual Meeting American Epilepsy Society. Disponível em: http://www.aesnet.org/go/publications/aes-abstracts/abstractsearch/mode/display/st/Politsky/sy/2010/sb/Authors/id/12296 